\begin{tabular}{|c|c|}
\hline Title & Nonlinear Carrier Dynamics in Semi-Metal Bismuth Induced by Intense Terahertz Field \\
\hline Author(s) & $\begin{array}{l}\text { A raki, Kotaro; Minami, Y asuo; Dao, Thang Duy; Nagao, Tadaaki; Takeda, Jun; Kitajima, Masahiro; Katay ama, } \\
\text { Ikufumi }\end{array}$ \\
\hline Citation & $\begin{array}{l}\text { International Conference on Ultrafast Phenomena } 2014 \text { Okinawa Japan 7-11 July } 2014 \text { ISBN:1-55752-279-0, paper } \\
\text { 09.W ed.P3.29 } \\
\text { https://doi.org/10.1364/NP.2014.09.W ed.P3.29 }\end{array}$ \\
\hline Issue Date & 2014 \\
\hline Doc URL & http:/hdl .handle.net/2115/72282 \\
\hline Type & proceedings \\
\hline File Information & UP_09_Wed_P3.pdf \\
\hline
\end{tabular}

Instructions for use 


\title{
Nonlinear Carrier Dynamics in Semi-Metal Bismuth Induced by Intense Terahertz Field
}

\author{
Kotaro Araki ${ }^{1}$, Yasuo Minami ${ }^{1}$, Thang Duy Dao ${ }^{2,3}$, Tadaaki Nagao ${ }^{2,3}$, \\ Jun Takeda ${ }^{1}$, Masahiro Kitajima ${ }^{1,3,4,5}$, and Ikufumi Katayama ${ }^{1}$ \\ ${ }^{1}$ Department of Physics, Faculty of Engineering, Yokohama National University, Yokohama 240-8501, Japan \\ ${ }^{2}$ International Center for Materials Nanoarchitectonics, National Institute for Materials Science, Tsukuba 305-0044, Japan \\ ${ }^{3}$ CREST, Japan Science and Technology Agency, Kawaguchi 332-0012, Japan \\ ${ }^{4}$ LxRay Co. Ltd., Nishinomiya 663-8172, Japan \\ ${ }^{5}$ Department of Applied Physics, National Defense Academy, Yokosuka 239-8686, Japan \\ E-mail address: katayama@ynu.ac.jp
}

\begin{abstract}
We investigated nonlinear carrier response of semi-metal bismuth under intense terahertz pulse illumination. By applying the intense terahertz field, the transmittance increases more than $10 \%$, indicating an increase of the effective mass.

OCIS codes: (300.6495) Spectroscopy, terahertz; (310.6860) Thin films; (320.7110) Ultrafast nonlinear optics
\end{abstract}

\section{Introduction}

Semi-metal bismuth (Bi) has been investigated over the years, and many interesting properties such as very small effective mass, high Hall coefficient, and non-parabolic band dispersion have been observed [1]. In addition, semimetal-to-semiconductor transition (SMSC) and structural phase transformation have been reported for the ultrathin Bi films below $30 \mathrm{~nm}$ and 1-2 $\mathrm{nm}$, respectively [2,3]. Therefore, the optical properties of Bi ultrathin film have been attracting attention. Recently, we have experimentally revealed the optical response of the surface state of Bi ultra-thin films by using broadband terahertz time domain spectroscopy (THz-TDS) [4]. In the present work, we have found an anomalous transmittance increase in the transmission spectrum in $\mathrm{Bi}$ film induced by intense $\mathrm{THz}$ pulses, revealing the nonlinear carrier response coming from the non-parabolic band structure of Bi.

\section{Experiment}

Single-crystalline $\mathrm{Bi}(001)$ film was deposited at room temperature on $\mathrm{Si}(111)-7 \times 7$ surface after the cleaning by $\mathrm{DC}$ resistive heating at $1500 \mathrm{~K}$ in an ultrahigh vacuum chamber. After the deposition, the sample was annealed at $350 \mathrm{~K}$ to obtain atomically flat film. The thickness of Bi film was $40 \mathrm{~nm}$. Details of the method were previously described elsewhere [5].

The experimental setup of THz-TDS is shown in Figure 1(a). The light source is a Ti-Sapphire regenerative amplifier with center wavelength of $800 \mathrm{~nm}$, pulse duration of $130 \mathrm{fs}$, repetition of $1 \mathrm{kHz}$, and pulse energy of 1.7 $\mathrm{mJ}$. The wavefront-tilted laser pulse satisfying the non-collinear phase matching condition of optical rectification in $\mathrm{MgO}: \mathrm{LiNbO} 3$ generates an intense single cycle terahertz pulse [6]. The maximum field strength of the terahertz pulse is more than $160 \mathrm{kV} / \mathrm{cm}$. The terahertz pulse is focused onto Bi thin film by off-axis parabolic mirrors, and then the transmitted terahertz pulse is detected by electro-optic (EO) sampling method using a 0.4-mm-thick GaP (110) crystal. All the measurements are performed at room temperature and under a nitrogen atmosphere to remove the influence of water vapor. Wire grid polarizers are used to adjust the intensity of the generated terahertz wave.

\section{Result and Discussion}

Figure 1(b) shows the maximum terahertz field dependence of the transmittance of Bi. When increasing the terahertz field up to $160 \mathrm{kV} / \mathrm{cm}$, transmittance increased by $10 \%$ or more compared with the low-field limit. The transmittance increases by $10 \%$ or more with increasing the terahertz field up to $160 \mathrm{kV} / \mathrm{cm}$. This result shows the anomalous non-linearity of the carrier response in $\mathrm{Bi}$. In order to explain this behavior, we analyzed the transmitted spectra using a Drude model. In this model, the complex dielectric constant is described as

$$
\widetilde{\varepsilon}(\omega)=\varepsilon_{\infty}-\frac{\omega_{p}{ }^{2}}{\omega(\omega+i \gamma)},
$$

where $\varepsilon_{\infty}$ is dielectric constant at high frequency limit, $\omega_{p}$ is the plasma frequency, and $\gamma$ is the damping constant, respectively. Here, the plasma frequency is expressed as 
(a)

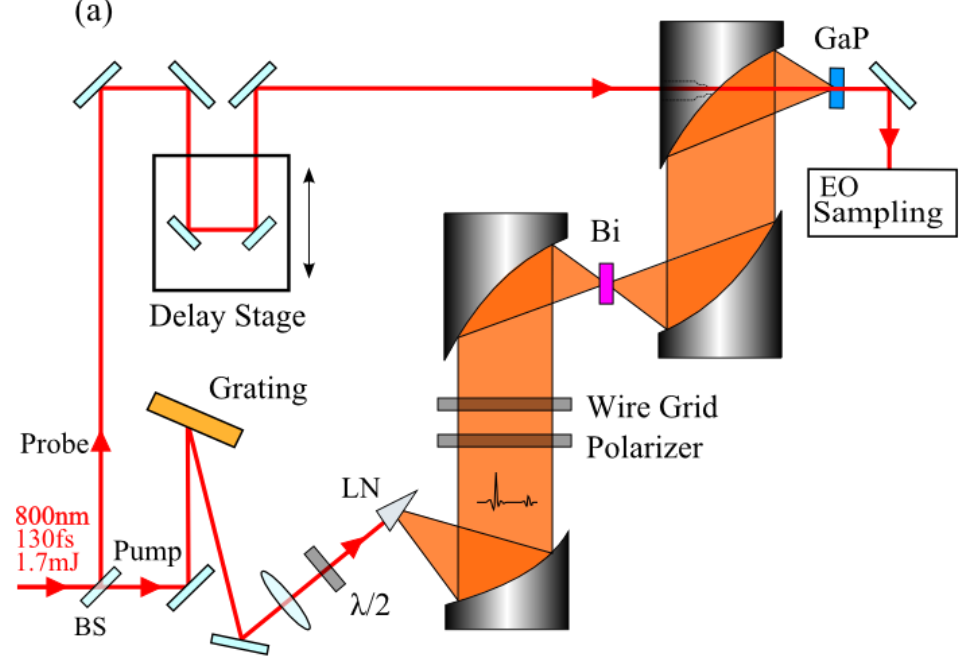

(b)

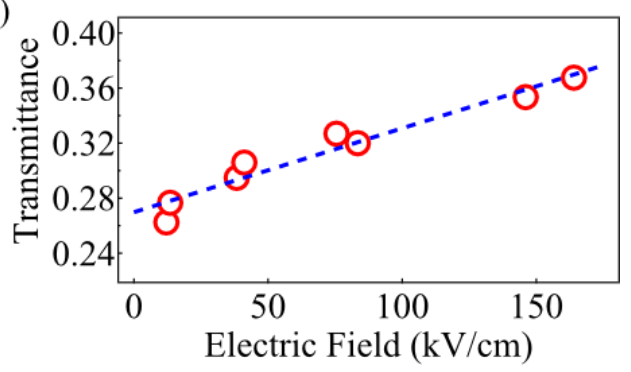

(c)

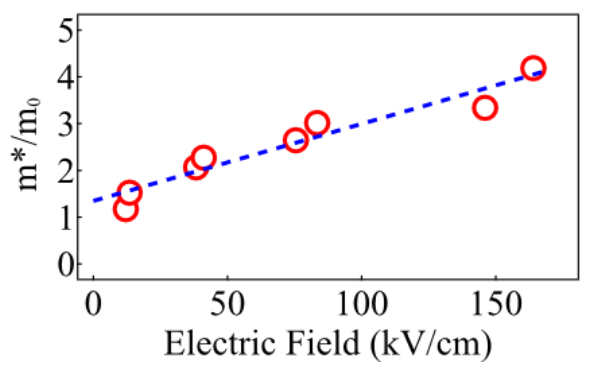

Fig. 1 (a) Schematic view of the experimental setup. Intense terahertz pulses are generated by the tilted pulse front excitation for $\mathrm{MgO}_{\mathrm{LiNbO}}$ (LN) and detected by EO sampling using 0.4-mm-thick GaP crystal. (b) Transmittance intensity as a function of the maximum electric field intensity. Open circle is experimental data, and a dashed line is the guide for eyes. (c) Maximum electric field intensity dependence of the effective mass of carriers normalized by the effective mass at the low-field limit $(<1 \mathrm{kV} / \mathrm{cm})$.

$$
\omega_{p}=\sqrt{\frac{n e^{2}}{\varepsilon_{0} m^{*}}},
$$

where $n$ is the carrier density, $m^{*}$ is the effective mass of the carriers, $e$ is the elementary charge of electrons, and $\varepsilon_{0}$ is the dielectric constant in a vacuum. Assuming that the carrier density is constant, the effective mass can be estimated from eqs. (1) and (2). Figure 1(c) shows the field intensity dependence of the effective mass normalized by that at low electric field limit. As shown in Figure 1(c), the effective mass dramatically increases more than $300 \%$ under the intense terahertz field illumination. This result indicates that the electrons at L-point of Bi are intensively moved to a nonlinear region. Because the band structure of electrons at L-point is similar to that of Dirac electrons, the electrons can easily be accelerated along the linear dispersion, leading to the anomalous nonlinear response.

\section{Conclusion}

We have investigated the electric field strength dependence of the terahertz transmittance of Bi film. Based on the Drude model analysis, we found the anomalous non-linear response of electrons whose effective mass dramatically increases. The result comes from characteristics of electrons with non-parabolic band dispersion in $\mathrm{Bi}$, which is analogous to that of Dirac electrons.

\section{References}

[1] Y. Liu and R. E. Allen, "Electronic structure of the semimetals Bi and Sb", Phys. Rev. B 52, 1566 (1995).

[2] C. A. Hoffman, J. R. Meyer, and F. J. Bartoli, “Semimetal-to-semiconductor transition in bismuth thin film”, Phys. Rev. B 48, 11431 (1993).

[3] T. Nagao, J. T. Sadowski, M. Saito, S. Yaginuma, Y. Fujikawa, T. Kogure, T. Ohno, Y. Hasegawa, S. Hasegawa, and T. Sakurai, "Nanofilm Allotrope and Phase Transformation of Ultrathin Bi Film on Si(111)-7×7”, Phys. Rev. Lett. 93, 105501 (2004).

[4] K. Yokota, J. Takeda, C. Dang, G. Han, D. N. McCarthy, T. Nagao, S. Hishita, M. Kitajima, and I. Katayama, "Surface metallic states in ultrathin Bi(001) films studied with terahertz time-domain spectroscopy”, Appl. Phys. Lett. 100, 251605 (2012).

[5] S. Yaginuma, K. Nagaoka, T. Nagao, G. Bihlmayer, Y. M. Koroteev, E. V. Chulkov, and T. Nakayama, "Electronic Structure of Ultrathin Bismuth Films with A7 and Black Phosphorus like Structures", J. Phys. Soc. Jpn. 77, 014701 (2008).

[6] J. Hebling, G. Almási, I. Z. Kozma, and J. Kuhl, "Velocity matching by pulse front tilting for large area THz-pulse generation", Opt. Express 10, 1161 (2002). 